\title{
Carrier Statistics and Quantum Capacitance Models of Graphene Nanoscroll
}

\author{
M. Khaledian, ${ }^{1}$ Razali Ismail, ${ }^{1}$ M. Saeidmanesh, ${ }^{1}$ M. T. Ahmadi, ${ }^{2}$ and E. Akbari ${ }^{3}$ \\ ${ }^{1}$ Department of Electronic Engineering, Faculty of Electrical Engineering, Universiti Teknologi Malaysia, \\ 81310 Skudai, Johor Bahru, Malaysia \\ ${ }^{2}$ Nanotechnology Research Center Nanoelectronic Group, Physics Department, Urmia University, Urmia 57147, Iran \\ ${ }^{3}$ Centre for Artificial Intelligence and Robotics (CAIRO), Universiti Teknologi Malaysia, 81300 Skudai, Johor Bahru, Malaysia
}

Correspondence should be addressed to Razali Ismail; razali@fke.utm.my

Received 20 January 2014; Accepted 8 April 2014; Published 12 June 2014

Academic Editor: Xuedong Bai

Copyright $\odot 2014$ M. Khaledian et al. This is an open access article distributed under the Creative Commons Attribution License, which permits unrestricted use, distribution, and reproduction in any medium, provided the original work is properly cited.

As a new category of quasi-one-dimensional materials, graphene nanoscroll (GNS) has captivated the researchers recently because of its exceptional electronic properties like having large carrier mobility. In addition, it is admitted that the scrolled configurations for graphene indicate larger stability concerning the energy, as opposed to their counterpart planar configurations like nanoribbon, nanotube, and bilayer graphene. By utilizing a novel analytical approach, the current paper introduces modeling of the density of state (DOS), carrier concentration, and quantum capacitance for graphene nanoscroll (suggested schematic perfect scroll-like Archimedes spiral). The DOS model was derived at first, while it was later applied to compute the carrier concentration and quantum capacitance model. Furthermore, the carrier concentration and quantum capacitance were modeled for both degenerate and nondegenerate regimes, along with examining the effect of structural parameters and chirality number on the density of state and carrier concentration. Latterly, the temperature effect on the quantum capacitance was studied too.

\section{Introduction}

It is well agreed that the graphene based materials do reveal better electrical transmission. Fabricating the electronic devices in tremendously small dimensions (fewer than 100 nanometers) has become possible owing to the modern advances in the construction of atomic-sized conductors. Up till now, the majority of surveys conducted formerly have focused on CNT and GNR [1,2]. It is known that the graphene nanoscrolls are indeed small graphene nanoribbons which have been rolled up into the spiral [3, 4]. The GNS formation mechanisms greatly resemble the mechanisms associated with the characteristic large graphene and boron nitride nanoscrolls. The overlapping surface of the rolled layers in GNSs is potent of enhancing the structural stability [5]. Graphene nanoscrolls are reported to be auspicious materials for the subsequent generation of the nanoelectronic devices, like the channel and interconnection in
FETs and MOSFETs $[6,7]$. While outlining the structure of the nanoscrolls, the electron microscopy and diffraction can be measured as very efficient approaches $[8,9]$. GNSs have the potential being used as electron-transport carrier $[10,11]$. Currently, the quasi-one-dimensional nanocarbons, namely, the nanowall, nanowire, nanobelt, and nanoscroll, are being synthesized using the hydrothermal method or the plasma enhanced chemical vapor deposition (CVD) [1214]. Utilizing the isotropy alcohol solution for rolling up the monolayer graphene predefined on $\mathrm{SiO}_{2} / \mathrm{Si}$ substrates has been considered as a simple and efficient method for constructing the high-quality carbon nanoscrolls (CNSs). Additionally, it is confirmed that the GNS attained throughout this method would be capable of sustaining a high current density up to $5 \times 10^{7} \mathrm{~A} / \mathrm{cm}^{2}$. This in turn proves that it can be taken as a proper candidate for microcircuit interconnect $[15,16]$. Another important class of nanostructures is boron nitride nanotubes (BNNTs) and boron nitride 
nanoscrolls (BNSs). Although a large number of theoretical and experimental works have been reported to BNNTs, BNSs have not been explored so far. In principle, the recently reported experimental techniques used to produce CNSs can be used to produce BNSs using cubic boron nitride crystals as starting materials. Similar to CNSs, BNS formation is dominated by two major energetic contributions, the elastic energy increase caused by bending the BN layer (decreasing stability) and the free energy decrease generated by the van der Waals interaction energy of overlapping regions of the layer (increasing stability). This suggests that the van der Waals interactions are more relevant for the BNS than for the CNS case. [17, 18]. Figure 1 illustrates a common graphene nanoscroll (perfect scroll-like Archimedes spiral) whose length $L$ (length of Archimedes spiral) is given by

$$
L=\int_{a}^{b} \sqrt{r^{2}+\left(\frac{d r}{d \theta}\right)^{2}} d \theta
$$

where $\theta$ stands as right angle for the spiral from $a$ to $b$ for $a$ as the starting value from $\theta, b=2 \pi z$, ( $z$ is the number of turns), and $r=a+b \theta$ ( $r$ is the distance from the origin). By utilizing a third nearest-neighbour tight binding analysis, the energy dispersion relation and conductance of graphene nanoscroll can be achieved. The dependency of the energy band gap on the geometry structure and chirality number has been ratified [19]. Therefore, the controllable band gap in GNS can stand as the foremost point in future studies for employing the GNS as electronic devices, like the FET and MOSFET. The current research presents modeling of the states density, the carrier concentration, and quantum capacitance on the basis of the parabolic energy dispersion approximation for the ZGNS. By implementing the first derivative of the Taylors expansion approximation on the energy dispersion relation, the quantum capacitance is accordingly modelled. Likewise, MaxwellBoltzmann's distribution law was used at low concentration. In the meantime, the quantum capacitance was only regarded to be within parabolic bands [20-22]. Besides, the carrier statistics dependency on the length of the spiral $(L)$ and the chiral vector $(\vec{c})$ was shown while examining the effects of the temperature variations on the quantum capacitance. In this paper, the effect of the electroactuation phenomenon on the model of the quantum capacitance has not been taken into account [23].

\section{Analytical Model of Quantum Capacitance $\left(C_{q}\right)$ for ZGNS}

As fundamental electronic parameters, the carrier concentration and quantum capacitance can aid in attaining the physical insights while specifying the electronic devices, like the FET and MOSFET characteristics [24, 25]. Numerous surveys have been conducted so far on the subject of the graphene, CNT, GNR, and GNSs electronic properties like the carrier statistics, conductance, mobility, and quantum capacitance through molecular dynamic simulation, as well as the fabrication process [26-28]. Nevertheless, there is no numerical simulation or analytical models for the stated

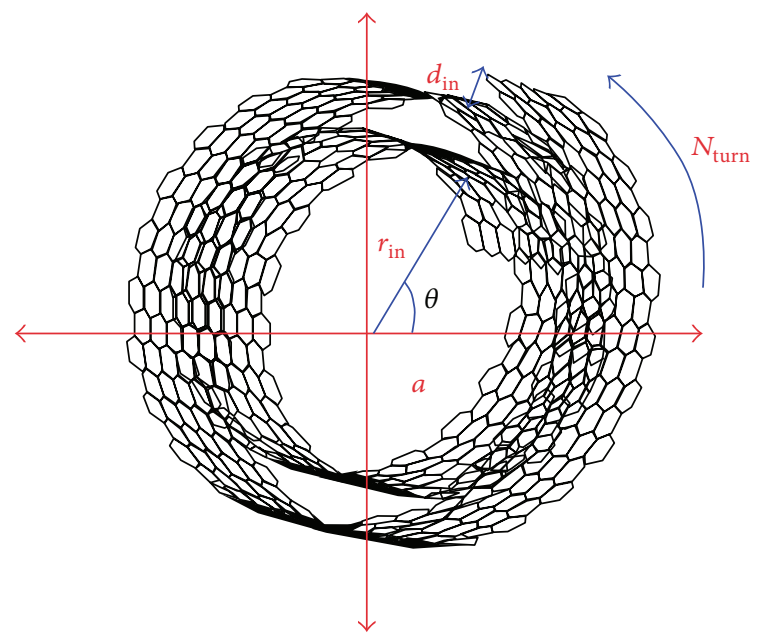

FIGURE 1: Schematic representation of a typical graphene nanoscroll.

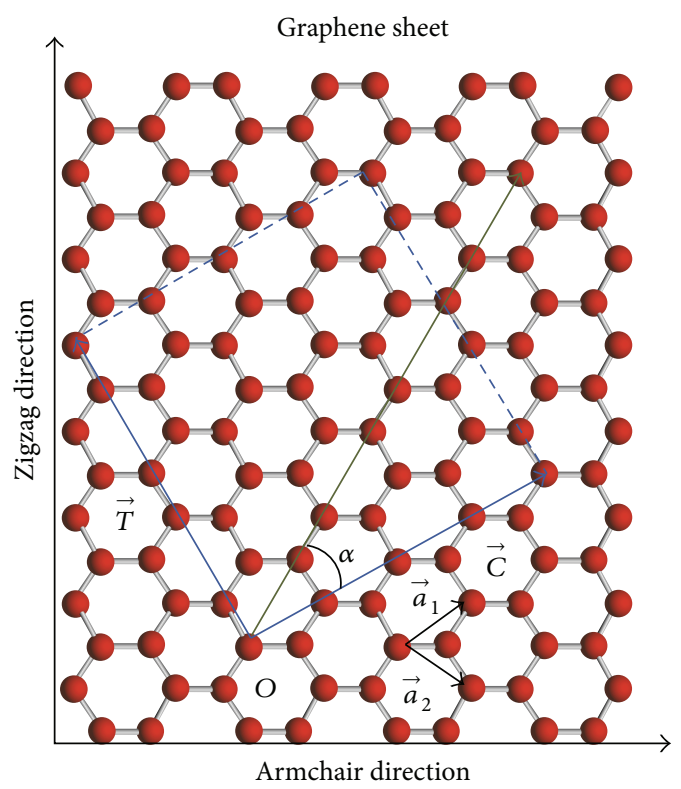

FIGURE 2: Illustration of how a graphene sheet is rolled up into spiral in GNS.

parameters in the GNSs. The graphene lattice presented in Figure 2 indicates the procedure of rolling the graphene layer up to the formation of GNS, in which $\alpha$ symbolizes the scroll angle pertaining to the axes. The chiral vector $(\vec{c})$ determines the direction of the roll-up $\vec{c}=n \vec{a}_{1}+m \vec{a}_{2}$, where $a_{1}\left(\sqrt{3} a_{c c}((\sqrt{3} / 2) \hat{i}+0.5 \hat{j})\right), a_{2}\left(\sqrt{3} a_{c c}((\sqrt{3} / 2) \hat{i}-0.5 \hat{j})\right)$ and where the integers $(n)$ and $(m)$ are the number of steps along the unit vectors $\vec{a}_{1}$ and $\vec{a}_{2}$. It is possible for us to have an armchair nanoscroll if $\vec{c}$ lies along the $x$-axis and a zigzag nanoscroll if $\vec{c}$ lies along the $y$-axis. Vector of $\vec{T}$ is the translational vector along the nanoscroll axis. [29-31]. Consequently, in zigzag graphene nanoscroll whose circumferential direction is around the $y$-axis, the energy dispersion relation utilizing 
the tight binding model and enforcing boundary condition $\vec{k} \cdot \vec{c}=L$ is determined as [19]

$$
E_{Z}(\vec{k})= \pm t \sqrt{1+4 \cos \left(\frac{3 k_{t} a_{c c}}{2}\right) \cos \left(\frac{L}{2 n}\right)+4 \cos ^{2}\left(\frac{L}{2 n}\right)},
$$

where $\vec{k}$ (the wave vector) can be obtained by $\vec{k}=k_{x} \hat{i}+k_{y} \hat{j}$, $k_{t}$ denotes the $k$ 's magnitude along the axis of the nanoscroll, and the range of $k_{t}$ will be $\left(-2 \pi / 3 a_{c c} \leq k_{t} \leq 2 \pi / 3 a_{c c}\right)$, while $a_{c c}=1.42 \AA$ will be the carbon-carbon bonding distance or the length of the carbon-carbon atom and $(t=$ $3.0 \mathrm{ev}$ ) stands as the closest neighbour $\mathrm{C}-\mathrm{C}$ overlap energy and $(n)$ signifies the chirality number. Figure 3 exhibits the energy band structure of ZGNS $E_{Z}(\vec{k})$ near the Dirac point with the band gap at $(k=0)$. Therefore, at the beginning for the modeling of quantum capacitance, we start by the modeling of the DOS which is presented for the zigzag nanoscroll. Because the structure of the ZGNS is a confined one-dimensional (1D) structure, the DOS (normalized per unit length) for ZGNS can be obtained through [24]

$$
\begin{aligned}
\operatorname{DoS} & =\frac{\Delta n_{i}}{L \Delta E} \\
& = \pm \frac{2 m^{*}\left(E-E_{c}\right)^{-1 / 2}}{3 \hbar^{2} \pi \sqrt{t}}, \\
m^{*} & =\frac{4 n^{2} \hbar^{2}}{9 a_{c c} t\left(L^{2}+4 n^{2}\right)},
\end{aligned}
$$

where $m^{*}$ provided by (4) can be considered as the efficient electron mass in the ZGNS, $(t=3.0 \mathrm{ev})$ is the closest neighbour $\mathrm{C}-\mathrm{C}$ overlap energy, $E_{c}$ denotes the energy of the conductance band derived as $E_{c}=0.5 t+\left(3 L^{2}\right) /\left(2 n^{2}\right)$, $L$ shows the length of the spiral in GNS, and $n$ stands for the chirality, while $\hbar$ signifies the reduced Planck's constant or Dirac constant. Figures 4 and 5 exhibit the DOS as a function of $E(k)$ for diverse values of $(n)$ and $(L)$. As it can be discerned, the DOS is proportional to $(L)$ and $(n)$ in reverse. In practice, by incorporating and integrating the FermiDirac distribution function against the energy, the sum of the carrier concentration within a band can be acquired. Accordingly, the carrier concentration can be demarcated as [20]

$$
n_{i}=\int \operatorname{DoS}(E) f(E) d E,
$$

where $f(E)=1 /\left(1+e^{\left(E-E_{F}\right) / K_{B} T}\right)$ stands as Fermi-Dirac distribution function which yields the probability of occupation of a state at any energy level. In this function, $E_{F}$ is the Fermi energy, $K_{B}$ denotes Boltzmann's constant, and $T$ shows the absolute temperature. By employing the DOS provided by (3) along with substituting $x=\left(E-E_{c}\right) / K_{B} T$ and $\eta_{F}=$ $\left(E_{F}-E_{c}\right) / K_{B} T$, the carrier concentration will read as

$$
n_{i}=\frac{2 m^{*}}{3 \hbar^{2} \pi} \sqrt{\frac{K_{B} T}{t}} \int_{0}^{+\infty} \frac{(x)^{-1 / 2}}{1+\exp \left(x-\eta_{F}\right)} d x .
$$

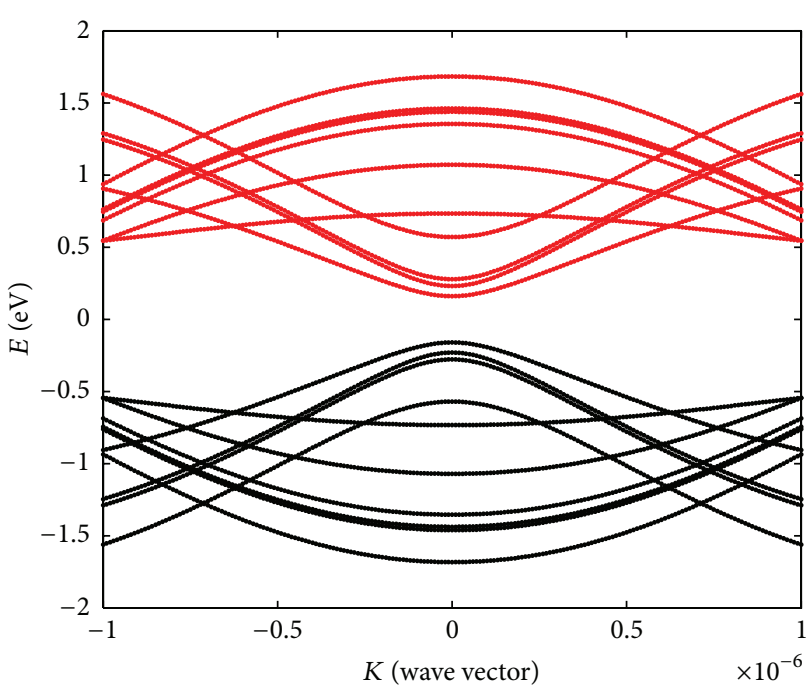

FIGURE 3: Energy band structure $(E)$ versus $(K)$ for ZGNS near the Dirac point.

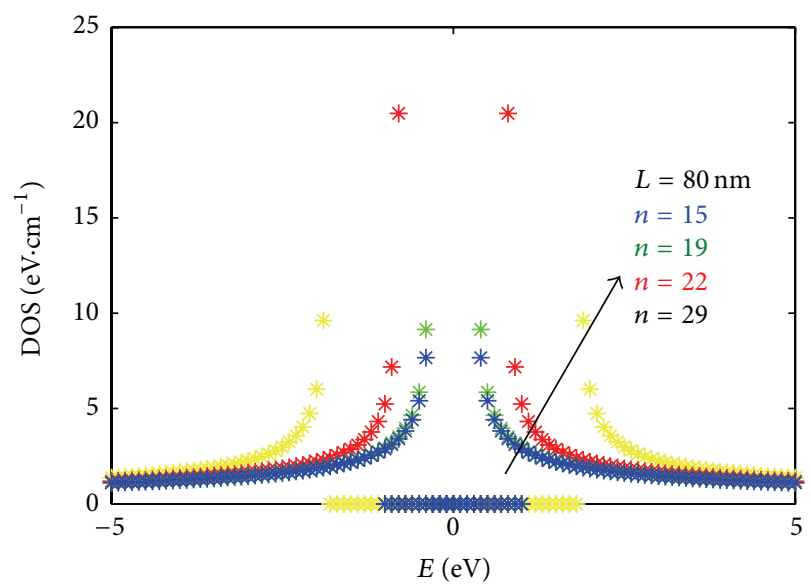

FIGURE 4: DOS versus $E$ for various values of chirality $(n)$ and $L=$ $80 \mathrm{~nm}$.

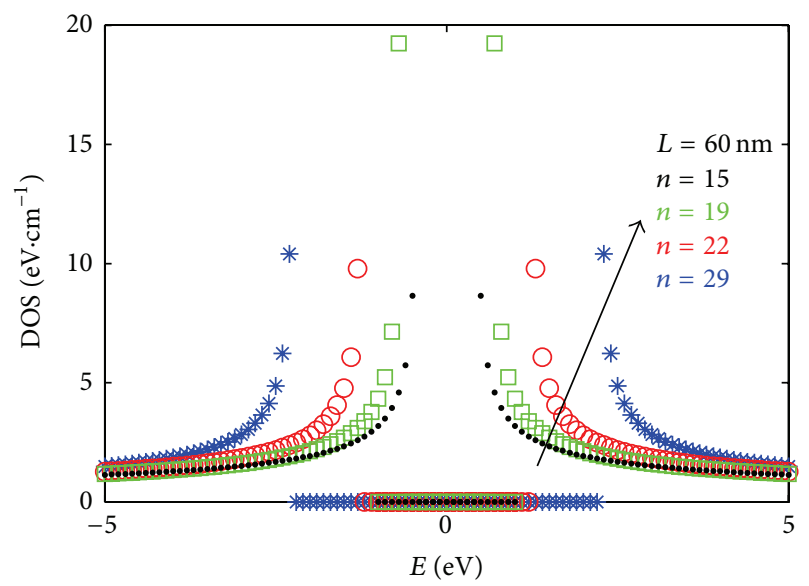

FIgURE 5: DOS versus $E$ for various values of chirality $(n)$ and $L=$ $60 \mathrm{~nm}$. 
By solving the integral analytically, (6) will be obtained as

$$
n_{i}=\frac{2 m^{*} K_{B} T}{3 t \pi \hbar^{2}} F_{0}\left(\eta_{F}\right)+\sqrt{\frac{2 m^{*} K_{B} T k^{2}}{9 t \pi \hbar^{2}}} F_{(-1 / 2)}\left(\eta_{F}\right),
$$

where $F_{0}\left(\eta_{F}\right)$ and $F_{(-1 / 2)}\left(\eta_{F}\right)$ show the Fermi-Dirac integrals of orders 0 and $(-1 / 2)$, respectively. Furthermore, by utilizing the equation of the energy dispersion, $k$ can be acquired as

$$
k= \pm \frac{1}{3} \sqrt{\frac{m^{*}\left(E-E_{c}\right)}{\hbar^{2} t}} .
$$

The carrier concentration as function of $\eta$ (normalized energy) for two diverse values of $L(L=30 \mathrm{~nm}, L=60 \mathrm{~nm}$, and $L=80 \mathrm{~nm}$ ) is plotted in Figure 6, indicating that, by a rise in $L$, there will be a rise in the $n_{i}$, while all of the curves in the figure would pursue the same trend. It should be noted that the Fermi-Dirac distribution function contains various forms in degenerate and nondegenerate states that can be attributed by $\left(\eta_{F} \gg 0\right)$ and $\left(\eta_{F} \ll 0\right)$, respectively $[32,33]$. As opposed to the $3 K_{B} T$, there are a small number of electrons in the conduction band in the nondegenerate regime while the conduction band edge is greatly above the Fermi energy. Therefore, the Fermi-Dirac integral can be estimated by the Maxwell-Boltzmann distribution factor of $f(E)=\exp \left(\eta_{F}\right)$. However, the concentration of electrons in the conductance band in the degenerate regime surpasses the state density, while the Fermi energy remains within the conductance band and the Fermi-Dirac function can be estimated as $f(E)=1$. Having implemented these limits for (7), the carrier concentration in degenerate and nondegenerate regimes can be, respectively, obtained by

$$
\begin{gathered}
n_{i \mathrm{dg}}=\frac{2 \eta_{F} m^{*} K_{B} T}{3 t \pi \hbar^{2}}+\frac{k}{t \pi \hbar} \sqrt{\frac{2 \eta_{F} m^{*} K_{B} T}{3}}, \\
n_{\text {inon-dg }}=\frac{2 m^{*} K_{B} T}{3 t \pi \hbar^{2}} \exp \left(\eta_{F}\right)+\sqrt{\frac{2 m^{*} K_{B} T k^{2}}{9 t \pi \hbar^{2}}} \exp \left(\eta_{F}\right) .
\end{gathered}
$$

Figure 7 demonstrates the carrier concentration with regard to $\eta$ for both degenerate and nondegenerate regimes. As it can be observed, the carrier concentration in the nondegenerate state is larger than the same concentration in the degenerate regime; moreover, by having a rise in the $\eta$, the $n_{i}$ correspondingly surges with a nonlinear relation. The quantum capacitance is remarkably influenced by the quantum confinement into nanoscale size devices. Consequently, the quantum capacitance has been supposed to be modeled for nanoscale devices, the graphene based materials in particular $[27,34]$. The quantum capacitance can be acquired by computing at a certain Fermi level and temperature, contrasting the total capacitance that is attained from the experiments. The quantum capacitance at Dirac point or charge neutrality is obtained by the differentiation of carrier concentration against the states of energy level. A general expression of the one-dimensional quantum capacitance is provided by $[21,26,34]$

$$
C_{q}=\frac{\partial Q}{\partial V}=\frac{e^{2} \partial n_{i}}{\partial E}=\frac{e^{2}}{K_{B} T} \frac{\partial n_{i}}{\partial x},
$$

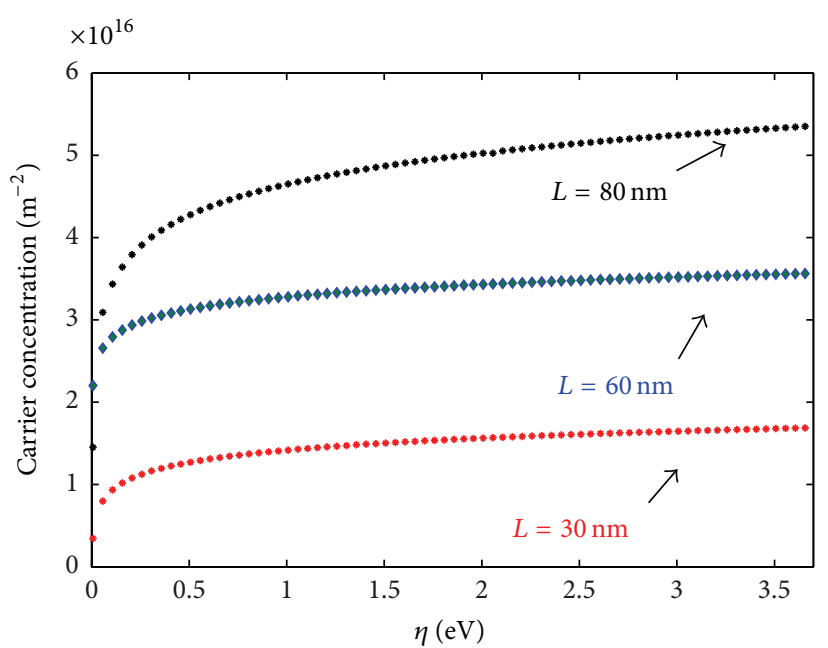

FIGURE 6: The carrier concentration with respect to $\eta$ for different values of $L$.

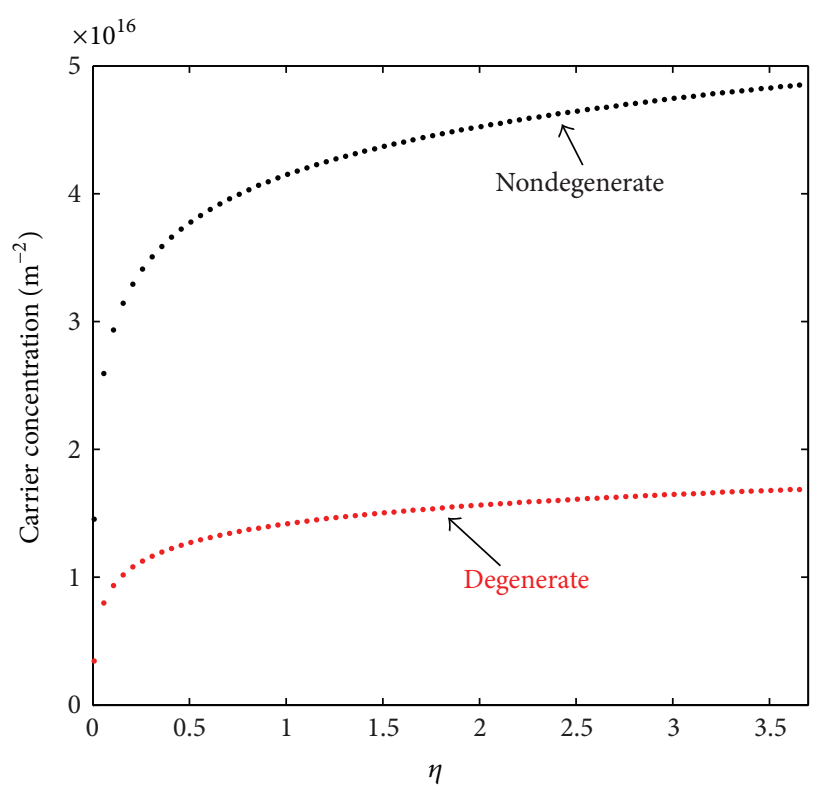

FIGURE 7: The carrier concentration with respect to $\eta$ for both degenerate and nondegenerate regimes.

where $n_{i}$ shows the carrier concentration and $(V)$ denotes the voltage applied to the GNS, while (e) stands as the magnitude of an electron charge. Consequently, by substituting $n_{i}$ from (7) into (10), we can simplify the quantum capacitance as

$$
C_{q}=\frac{2 e^{2} m^{*}}{3 \pi \hbar^{2}} \sqrt{\frac{1}{t \cdot K_{B} T}}\left(\frac{(x)^{-1 / 2}}{1+\exp \left(x-\eta_{F}\right)}\right) .
$$

The states within the conduction band (allowed band) are moderately filled at room temperature $(T=300 \mathrm{~K})$ in the nondegenerate regime; accordingly, the exponential part of (11) is large enough to discard (1) from the denominator. 
Therefore, the quantum capacitance in nondegenerate regime can be acquired as

$$
C_{q_{n} d}=\frac{2 e^{2} m^{*}}{3 \pi \hbar^{2}} \cdot(x)^{-1 / 2} \sqrt{\frac{1}{t \cdot K_{B} T}} \exp (x-\eta) .
$$

On the other hand, the exponential part is really small in the degenerate regime owing to the probability for the electron to fill all the available states up to the Fermi level which is roughly (1), suggesting that there are no accessible states within the conduction band for $E-E_{F} \leq 3 K_{B} T$. As a result, the quantum capacitance within the degenerate regime can be obtained by

$$
C_{q_{d}}=\frac{2 e^{2} m^{*}}{3 \pi \hbar^{2}} \sqrt{\frac{1}{t \cdot K_{B} T}} \cdot(x)^{-1 / 2} .
$$

The quantum capacitances against $\eta$ (normalized energy) for the general state and both degenerate and nondegenerate regimes are plotted in Figure 8, indicating that, for the degenerate regime, it has not been influenced by $\eta$ variations while it tends to have a steady value $\left(5.5 \times 10^{-9}\right)$. Contrariwise, it increases exponentially in the nondegenerate regime by altering the $\eta$. As well, the quantum capacitance in the general state upsurges intensely for the range of $1.5 \leq \eta \leq 5$; yet, it will reach the saturation region after that having the equal value as the degenerate limit. The dominance of the quantum capacitance can be explained at different concentration levels.

In the last part, the temperature effect on the quantum capacitance was scrutinized, showing that in Figure 9 the quantum capacitance gets to the degenerate limit more speedily once there is a rise in the temperature. Hence, the quantum capacitance, by elevating the temperature, will get to its limit at low $\eta$. There will be a considerable increase in the electron concentration by the temperature increase, making the quantum capacitance reach its limit more quickly, as a consequence. In the degenerate regime, the quantum capacitance is not supposedly influenced by the temperature by reason of the assumption of the high carrier density [25, 35]. Figure 9 portrays the quantum capacitance dependency in the general state for ZGNS on the temperature variations.

\section{Conclusion}

It is acknowledged that the graphene nanoscrolls with outstanding field emission properties and strip morphology are claimed to be capable of being employed in the future nanoelectronics as the electron transport carriers. Numerous analytical models have been recommended in the current paper for the GNSs electronic properties, such as the state density and the carrier concentration as well as the quantum capacitance. Furthermore, the current research approved that the chirality number $(n)$ and structural parameters of nanoscroll, such as length of nanoscroll $(L)$, affect the state density of states and carrier concentration. It is confirmed here that the results are consistent with the ones related to the carbon nanotube and graphene nanoribbon. Besides, the temperature effects on the quantum capacitance were examined, indicating that once there is a rise in the temperature,

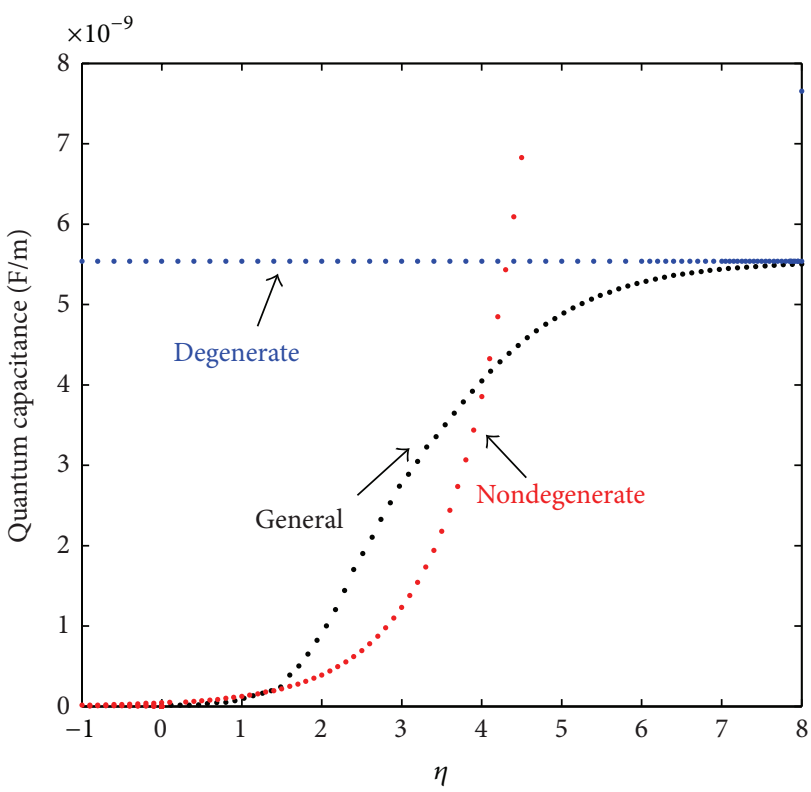

FIGURE 8: The quantum capacitance for both degenerate and nondegenerate regimes based on quantum capacitance in general state with respect to $\eta$ for ZGNS.

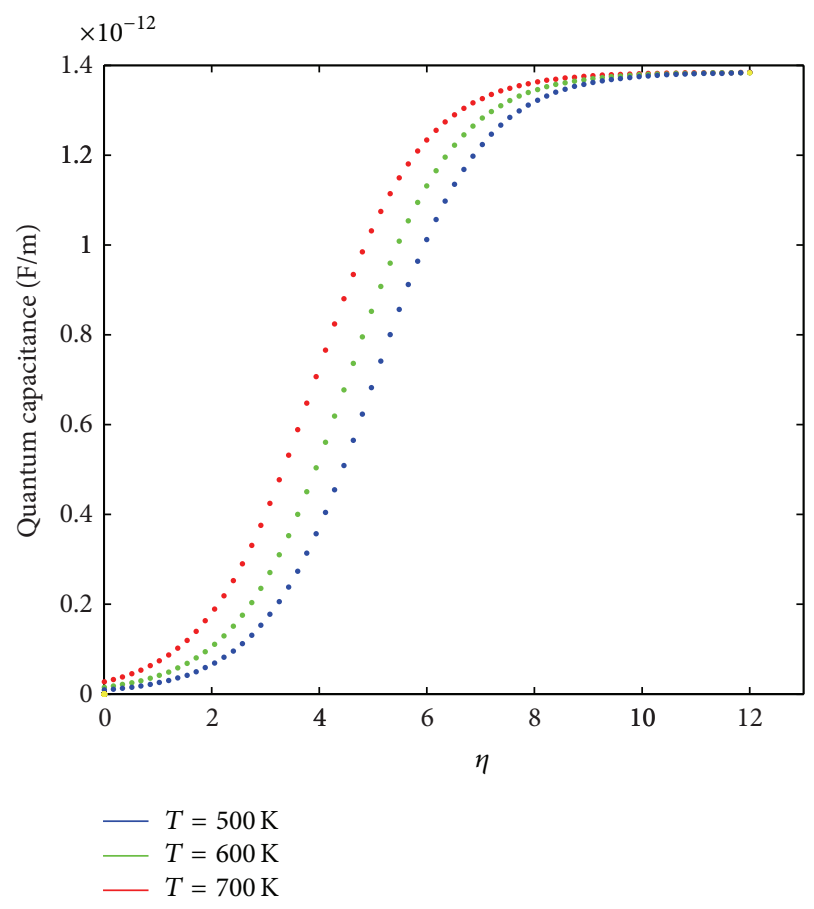

FIGURE 9: The effect of temperature on quantum capacitance in general state versus $\eta$ for ZGNS.

the quantum capacitance will reach its limit more quickly at low $\eta$.

\section{Conflict of Interests}

The authors declare that there is no conflict of interests regarding the publication of this paper. 


\section{Acknowledgments}

The authors would like to acknowledge the financial support from the Ministry of Higher Education (MOHE), Malaysia, under the Fundamental Research Grant Scheme (FRGS) Project no. R.J130000.7823.4F146. Also, thanks are due to the Research Management Centre (RMC) of Universiti Teknologi Malaysia (UTM) for providing excellent research environment to complete this work.

\section{References}

[1] M. Grundmann, "Nanoscroll formation from strained layer heterostructures," Applied Physics Letters, vol. 83, no. 12, pp. 2444-2446, 2003.

[2] S. F. Braga, V. R. Coluci, S. B. Legoas, R. Giro, D. S. Galvão, and R. H. Baughman, "Structure and dynamics of carbon nanoscrolls," Nano Letters, vol. 4, no. 5, pp. 881-884, 2004.

[3] B. V. C. Martins and D. S. Galvão, "Curved graphene nanoribbons: structure and dynamics of carbon nanobelts," Nanotechnology, vol. 21, no. 7, Article ID 075710, 2010.

[4] Y. Chen, J. Lu, and Z. Gao, "Structural and electronic study of nanoscrolls rolled up by a single graphene sheet," Journal of Physical Chemistry C, vol. 111, no. 4, pp. 1625-1630, 2007.

[5] X. Peng, J. Zhou, W. Wang, and D. Cao, "Computer simulation for storage of methane and capture of carbon dioxide in carbon nanoscrolls by expansion of interlayer spacing," Carbon, vol. 48, no. 13, pp. 3760-3768, 2010.

[6] A. K. Schaper, H. Hou, M. Wang, Y. Bando, and D. Golberg, "Observations of the electrical behaviour of catalytically grown scrolled graphene," Carbon, vol. 49, no. 6, pp. 1821-1828, 2011.

[7] X. Xie, L. Ju, X. Feng et al., "Controlled fabrication of highquality carbon nanoscrolls from monolayer graphene," Nano Letters, vol. 9, no. 7, pp. 2565-2570, 2009.

[8] G. Xi, M. Zhang, D. Ma, Y. Zhu, H. Zhang, and Y. Qian, "Controlled synthesis of carbon nanocables and branchednanobelts," Carbon, vol. 44, no. 4, pp. 734-741, 2006.

[9] Y.-X. Qi, M.-S. Li, and Y.-J. Bai, "Carbon nanobelts synthesized via chemical metathesis route," Materials Letters, vol. 61, no. 4-5, pp. 1122-1124, 2007.

[10] X. Shi, N. M. Pugno, and H. Gao, "Mechanics of carbon nanoscrolls: a review," Acta Mechanica Solida Sinica, vol. 23, no. 6, pp. 484-497, 2010.

[11] K. S. Kim, Y. Zhao, H. Jang et al., "Large-scale pattern growth of graphene films for stretchable transparent electrodes," Nature, vol. 457, no. 7230, pp. 706-710, 2009.

[12] G. Mpourmpakis, E. Tylianakis, and G. E. Froudakis, "Carbon nanoscrolls: a promising material for hydrogen storage," Nano Letters, vol. 7, no. 7, pp. 1893-1897, 2007.

[13] E. Perim and D. S. Galvao, "The structure and dynamics of boron nitride nanoscrolls," Nanotechnology, vol. 20, no. 33, Article ID 335702, 2009.

[14] A. Cao, G. Meng, and P. M. Ajayan, "Nanobelt-templated growth of carbon nanotube rows," Advanced Materials, vol. 16, no. 1, pp. 40-44, 2004.

[15] L. H. Viculis, J. J. Mack, and R. B. Kaner, "A chemical route to carbon nanoscrolls," Science, vol. 299, no. 5611, p. 1361, 2003.

[16] D. Xia, J. Xie, H. Chen et al., "Fabrication of carbon nanoscrolls from monolayer graphene," Small, vol. 6, no. 18, pp. 2010-2019, 2010.
[17] X. Li, X. Hao, M. Zhao et al., "Exfoliation of hexagonal boron nitride by molten hydroxides," Advanced Materials, vol. 25, no. 15, pp. 2200-2204, 2013.

[18] E. Perim, R. Paupitz, and D. S. Galvão, "Controlled route to the fabrication of carbon and boron nitride nanoscrolls: a molecular dynamics investigation," Journal of Applied Physics, vol. 113, no. 5, Article ID 054306, 2013.

[19] M. Khaledian, M. T. Ahmadi, R. Ismail, and M. Saeidmanesh, "Structural and properties of graphene nanobelts rolled up into spiral by a single graphene sheet," Journal of Computational and Theoretical Nanoscience, vol. 11, no. 3, pp. 601-606.

[20] J. Liang, D. Akinwande, and H.-S. P. Wong, "Carrier density and quantum capacitance for semiconducting carbon nanotubes," Journal of Applied Physics, vol. 104, no. 6, Article ID 064515, 2008.

[21] M. T. Ahmadi, J. F. Webb, N. A. Amin et al., "Carbon nanotube capacitance model in degenerate and nondegenerate regimes," AIP Conference Proceedings, vol. 1337, pp. 173-176, 2011.

[22] M. Lundstrom and J. Guo, Nanoscale Transistors: Device Physics, Modeling and Simulation, Springer, 1st edition, 2005.

[23] R. Rurali, V. R. Coluci, and D. S. Galvão, "Prediction of giant electroactuation for papyruslike carbon nanoscroll structures: first-principles calculations," Physical Review B: Condensed Matter and Materials Physics, vol. 74, no. 8, Article ID 085414, 2006.

[24] M. Lundstrom and J. Guo, "Basic concept," in Nanoscale Transistors: Device Physics, Modeling and Simulation, pp. 1-50, Springer Science+Business Media, Inc., New York, NY, USA, 2006.

[25] G. S. Kliros, "Quantum capacitance of bilayer graphene," in Proceedings of the 2010 33rd International Semiconductor Conference (CAS '10), vol. 1, pp. 69-72, October 2010.

[26] V. Parkash and A. K. Goel, "Quantum capacitance extraction for carbon nanotube interconnects," in Proceedings of the 20th International Conference on Microelectronics (ICM '08), pp. 292295, December 2008.

[27] T. Fang, A. Konar, H. Xing, and D. Jena, "Carrier statistics and quantum capacitance of graphene sheets and ribbons," Applied Physics Letters, vol. 91, no. 9, Article ID 092109, 2007.

[28] J. Xia, F. Chen, J. Li, and N. Tao, "Measurement of the quantum capacitance of graphene," Nature Nanotechnology, vol. 4, no. 8, pp. 505-509, 2009.

[29] P. R. Wallace, “The band theory of graphite," Physical Review, vol. 71, no. 9, pp. 622-634, 1947.

[30] M. T. Ahmadi, J. Frank Webb, R. Ismail, and M. Rahmandoust, "Carbon-based materials concepts and basic physics," in Advanced Nanoelectronics, pp. 65-98, 2012.

[31] S. Datta, Quantum Transport: Atom to Transistor, Cambridge University Press, 2nd edition, 2005.

[32] M. T. Ahmadi, Z. Johari, D. C. Y. Chek, N. A. Amin, and R. Ismail, "Modelling of graphene nanoribbon Fermi energy," Journal of Nanomaterials, vol. 2010, Article ID 909347, 6 pages, 2010.

[33] R. B. Dingle, Asymptotic Expansions: Their Derivation and Interpretation, Academic Press, 1973.

[34] L. Wei, D. J. Frank, L. Chang, and H.-S. P. Wong, "Noniterative compact modeling for intrinsic carbon-nanotube FETs: quantum capacitance and ballistic transport," IEEE Transactions on Electron Devices, vol. 58, no. 8, pp. 2456-2465, 2011.

[35] A. Hamzah, M. T. Ahmadi, and R. Ismail, "Quantum capacitance effect on zig-zag graphene nanoscrolls (ZGNS) (16,0)," Modern Physics Letters B, vol. 27, no. 1, Article ID 1350002, 2013. 

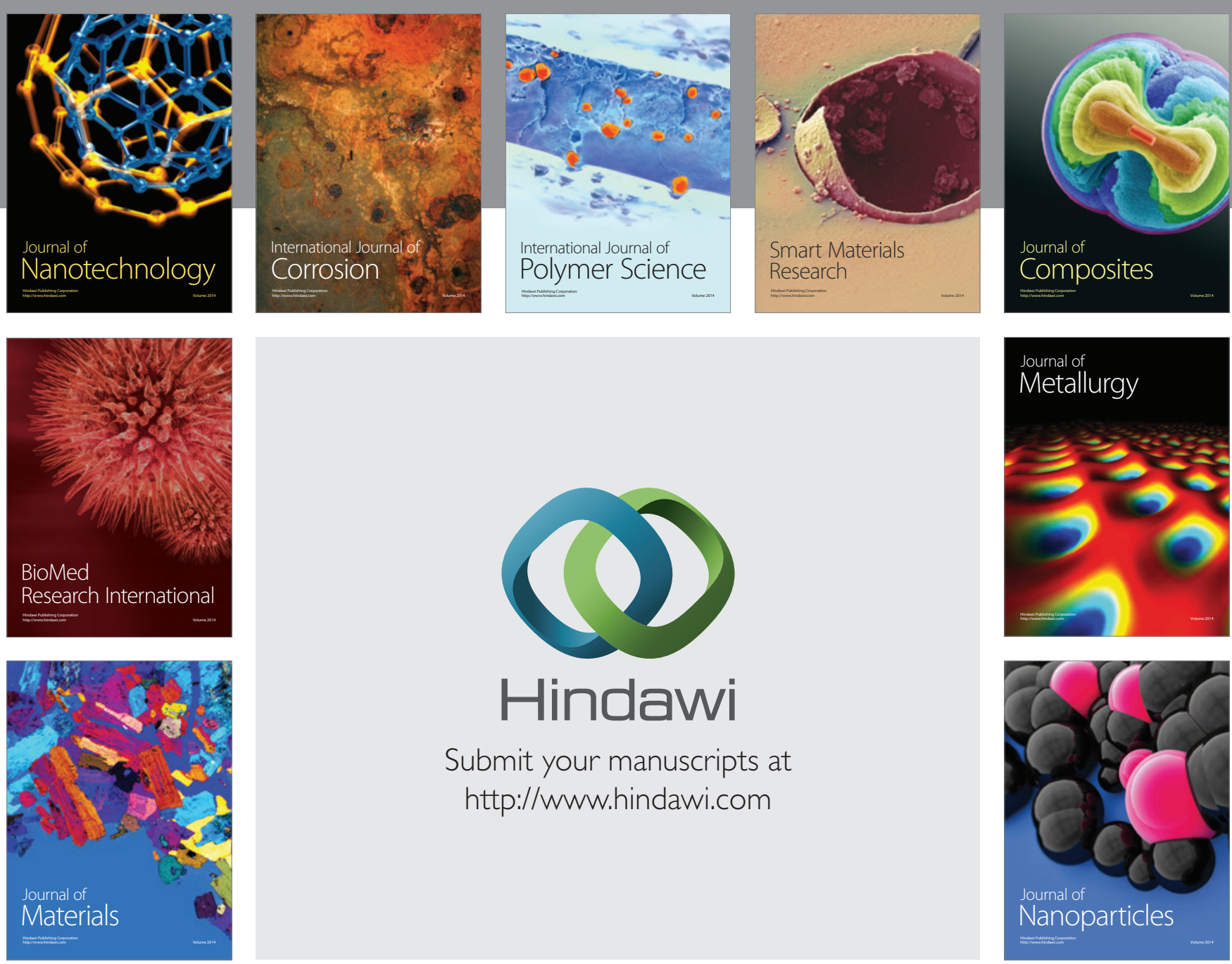

Submit your manuscripts at http://www.hindawi.com
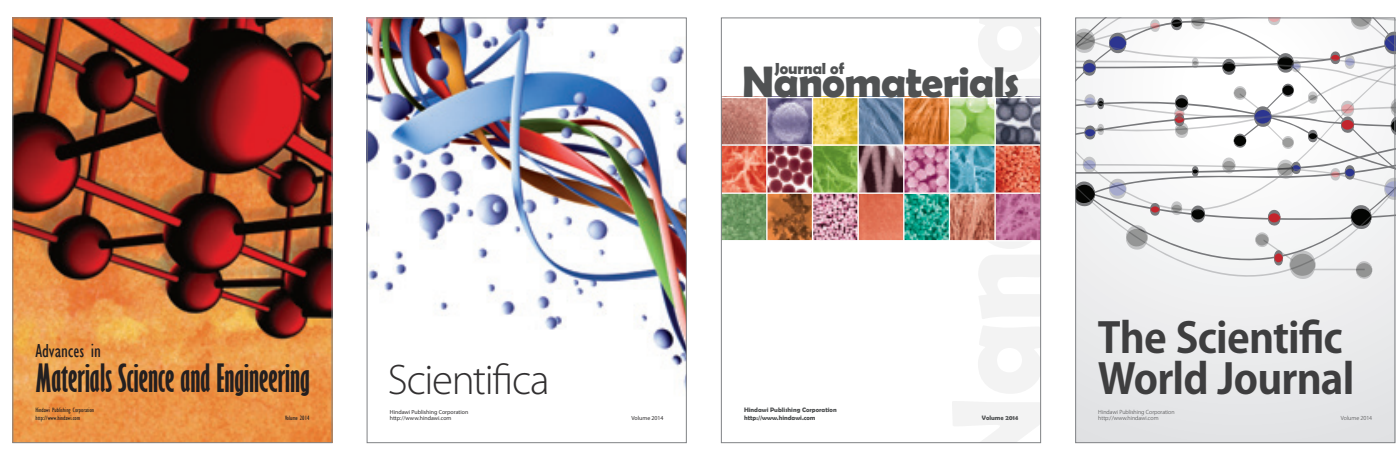

\section{The Scientific World Journal}
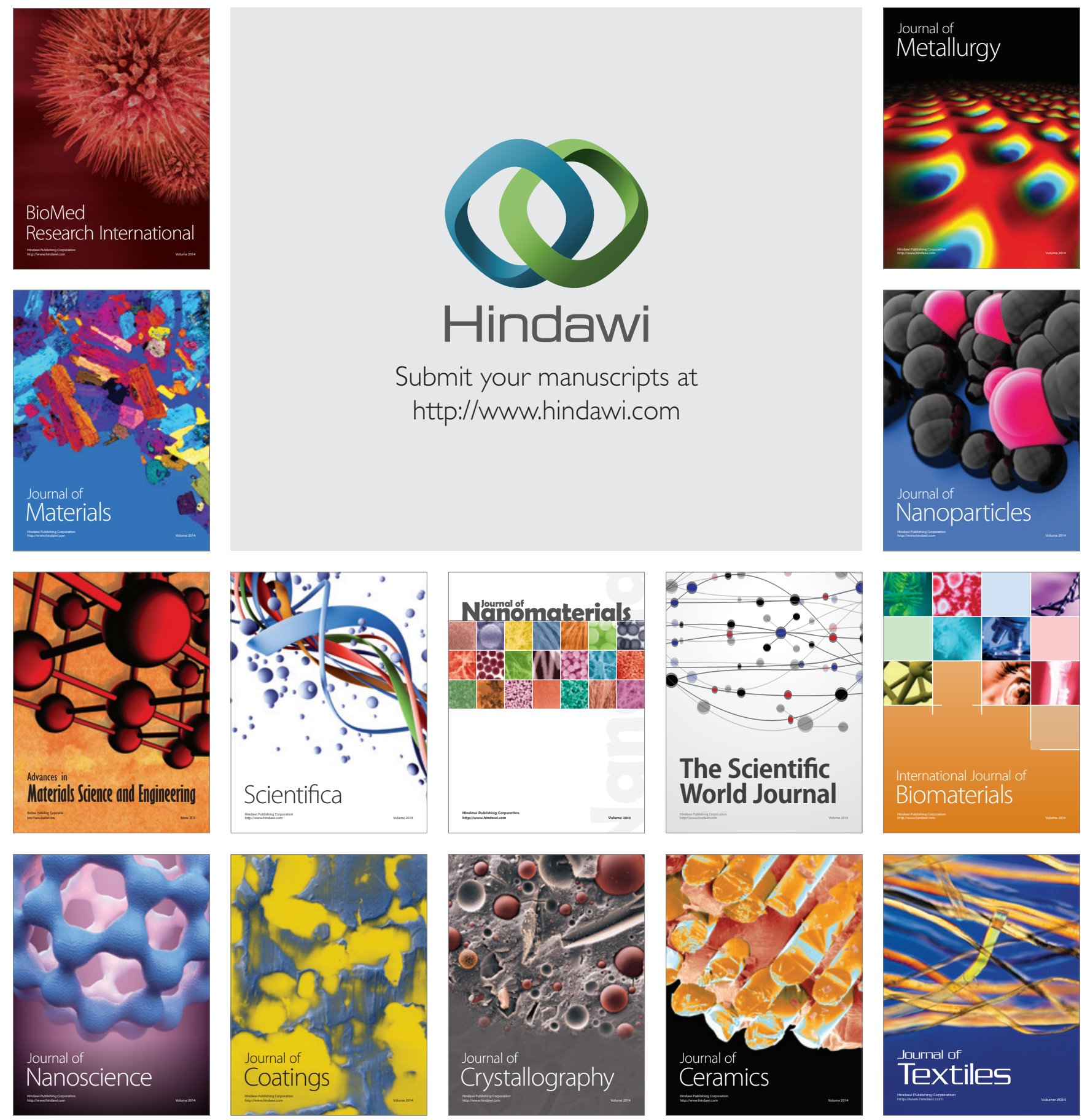